\title{
EUGENOL AMELIORATES PATHOGENIC FEATURES IN EXPERIMENTAL MODEL OF NAFLD IN RATS
}

BY

Maryam Hany Fawzi Azab Prof. Dr. Abtahal El Demerdash Zaki Dr. Noha Mohamed Said Abdel Azim Dr. Doaa Mokhtar El Sherbiny

\section{FROM}

Demonstrator, Department of Drugs and Toxicology, Faculty of Pharmacy, EgyptianRussian University

Professor and Head of Drugs and Toxicology Department, Faculty of Pharmacy, Ain Shams University

Lecturer, Department of Drugs and Toxicology, Faculty of Pharmacy, Egyptian-Russian University

Lecturer, Department of Drugs and Toxicology, Faculty of Pharmacy, Ain Shams University

\begin{abstract}
:
Background: Nonalcoholic fatty liver disease (NAFLD) is emerging one of the major causes of chronic liver damage that includes a wide spectrum of liver injury ranging from steatosis to steatohepatitis evolving to fibrosis.

Aim: This study was designed to evaluate the possible effect of EUG on NAFLD induced by high fat cholesterol diet.

Methods: Rats were fed either normal rat chow diet (control) or high fat cholesterol diet and both received olive oil $(10 \mathrm{mg} / \mathrm{kg}$ ) for eight weeks to induce NAFLD model. Eugenol $(10 \mathrm{mg} / \mathrm{kg})$ was administrated to rats by oral intubation 3 times weekly for 8 weeks.

Results: Our results showed that EUG significantly ameliorated the histopathological lesions induced by HFCD. Furthermore, HFCD induced a significant elevation in liver enzymes (alanine aminotransferase (ALT) and aspartate aminotransferase (AST)). This was significantly attenuated by EUG co-treatment.
\end{abstract}

Conclusion: These findings indicate that EUG possess es a marked role in modulation of NAFLD.

\section{Key words: eugenol; NAFLD.}

\section{Introduction:}

Non-alcoholic fatty liver disease (NAFLD) is the hepatic manifestation of the metabolic syndrome. It represents a spectrum of disease, ranging from simple steatosis to steatohepatitis through to fibrosis and cirrhosis (Than and Newsome, 2015). Currently, NAFLD is recognized as the most common form of liver disease worldwide affecting between $25-30 \%$ of the general population. Frequently, it has been associated with obesity, type 2 diabetes mellitus, hyperlipidemia, and insulin resistance (Berlanga et al., 2014). 
NAFLD hallmark is the excessive hepatic accumulation that results from an imbalance between lipid availability and lipid removal (Arguello et al., 2015). Once the energy expenditure is less than energy intake, lipid overload can be found in organs such as adipose tissue organs (Shulman, 2014) . As a consequence of excess lipid contents that spill over from the dysfunctional adipose tissue, ectopic lipid deposition in liver would occur (Stienstra et al., 2010). This flux in particular is a major determinant of accumulation of hepatic and lipoprotein fat in NAFLD that induce marked liver injury (Loria et al., 2008).

The effective therapy for NAFLD has not been established, but there are many proposed strategies and agents used for liver support (Yogalakshmi et al., 2010). Nowadays, renewed attention to alternative medicines and natural therapies has stimulated new wave of research to look for more efficacious agents with lesser side effects. Eugenol (4-allyl-2-methoxyphenol) is a naturally occurring phenolic compound extracted from clove, basil, cinnamon and nutmeg (Yogalakshmi et al., 2010). It has been used as: a fragrant and favoring agent (Nuchuchua et al., 2009), in dentistry as a filling material and a pulp capping agent or as a neuroprotective (Kabuto et al., 2007) and antibacterial (Kalemba and Kunicka, 2003). The hepatoprotective potentials of eugenol include antiinflammatory, antioxidant (Jirovetz et al., 2006) and anti-hyperglycemic effects (Srinivasan et al., 2014). The present study was designed to evaluate the possible protective effect of EUG on NAFLD induced by high fat cholesterol diet (HFCD).

\section{Materials and methods:}

\section{Drugs and chemicals:}

Eugenol was purchased from Sigma Chemical Co. (USA) and dissolved in olive oil. Cholesterol, casein, and methionine were purchased from Medico pharm Co., for Pharmaceutical and Medicine (Cairo, Egypt). Vitamin mixture and mineral mixture were obtained from Misr feed additives company (Egypt). All other chemicals were of the highest available commercial grade.

\section{Animals:}

Forty male albino rats weighed 150-180 g were obtained from Nile Co. for pharmaceutical and chemical industries (Egypt). The normal chow was acquired from Meladco for Animal Food (Egypt). Pellets and tap water were provided ad libitum. Temperature was maintained at $25^{\circ} \mathrm{C}$. Rats were allowed at least 1 week to acclimatize to the lab conditions. The study protocol was approved by the Ethical Committee, Faculty of Pharmacy, Ain Shams University, Egypt.

\section{Experimental design:}

White albino rats were randomly divided into four groups $(n=10)$ : group (1) fed normal rat chow diet and given olive oil $(10 \mathrm{mg} / \mathrm{kg})$ orally 3 times weekly for 8 weeks and is considered as control group. Group (2) fed HFCD diet and given olive oil (10 mg/kg) orally 3 times weekly for 8 weeks and is considered as HFCD group. Group (3) fed highHFCD diet and given EUG $(10 \mathrm{mg} / \mathrm{kg})$ orally 3 times weekly for 8 weeks and is considered 
as HFCD+EUG group. Group (4) fed normal rat chow diet and given EUG (10mg/kg) orally 3 times weekly for 8 weeks and is considered as EUG only group.

The high-fat high-cholesterol diet contained per $100 \mathrm{~g}, 58 \mathrm{gm}$ rat show diet, $5 \mathrm{gm}$ sucrose, $18 \mathrm{gm}$ butter, $10 \mathrm{gm}$ corn oil, $1 \mathrm{gm}$ cholesterol, $5 \mathrm{gm}$ casein, $0.2 \mathrm{gm}$ methionine, $0.8 \mathrm{gm}$ vitamins (vitamin mixture), and $2 \mathrm{gm}$ minerals (mineral mixture).

After 8 weeks, fasting blood samples were collected from the retro orbital venous plexus, allowed to clot and serum was separated by centrifugation at $3000 \mathrm{~g}$ for $15 \mathrm{~min}$ for biochemical assessment. Rats were then sacrificed; liver tissues were dissected out and washed with ice-cold saline. Then, parts from liver were fixed in $10 \%$ buffered formaldehyde for histopathological examination.

\section{Biochemical analysis:}

Serums alanine aminotransferase (ALT) and aspartate aminotransferase (AST), were determined by spectrophotometric method using commercially available kits (Teco Diagnostics, C.A., USA), and the results were expressed as IU/L.

\section{Histopathological examination}

For light microscopy, liver specimens were taken from the different lobes and fixed in $10 \%$ formalin and processed for paraffin sections of $4 \mu \mathrm{m}$ thickness. Sections were stained with hematoxylin and eosin (H \& E) and evaluated under light microscope.

\section{Statistical analysis}

All data are expressed as mean \pm SD. Statistical analysis differences among groups were analyzed by one-way analysis of variance (ANOVA) followed by Tukey Kramer test as a post hoc test. Differences were considered significant at $p<0.05$. All analyses were performed using lnstat version 3 software package. Graphs were sketched using Graph Pad prism (ISI ${ }^{\circ}$ software, USA) version 5 software.

\section{Results}

\section{Effect of Eugenol on liver index and liver enzymes}

Body weight was monitored throughout the feeding period. After 8 weeks, there was significant increase in liver index ratio in HFCD group by $86 \%$ as compared to the control group. On the contrary, co-treatment with EUG significantly decreased the liver index by $30 \%$ as compared to HFCD group (Fig.1). Similarly, ALT and AST significantly increased in HFCD group by 3 folds as compared to the control group. EUG co-treated rats showed significant decrease in ALT and AST by 65 and 62\%, respectively, as compared to HFCD group (Fig.2), (Fig 3), respectively. 
Figure 1: Effect of EUG on liver index in rats fed HFCD :

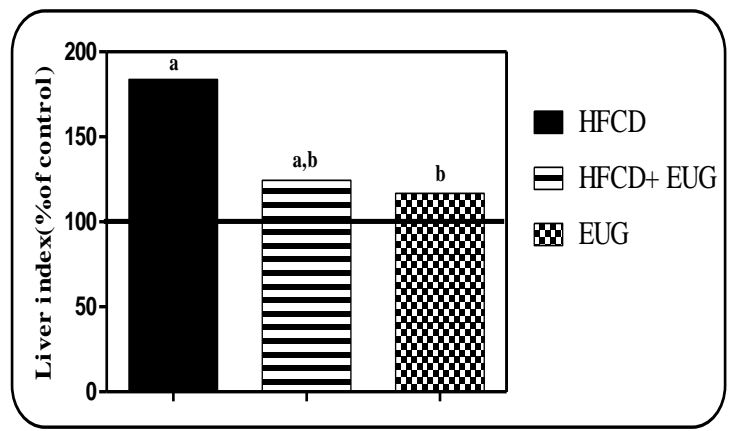

Fig (1).Effect of EUG on liver index in rats fed HFCD.

Rats were fed HFCD diet for 8 weeks, HFCD+ EUG, and EUG groups received EUG $(10 \mathrm{mg} / \mathrm{kg})$ orally 3 times weekly for 8 weeks .Data are represented as mean \pm S.D. $(\mathrm{n}=10)$. $\mathrm{a}$, or $\mathrm{b}$ : significant difference from control, or HFCD groups, respectively at $\mathrm{p}<0.05$ using ANOVA followed by Tukey-Kramer as a post-hoc test.

Figure 2: Effect of EUG on serum level of ALT in rats fed HFCD:

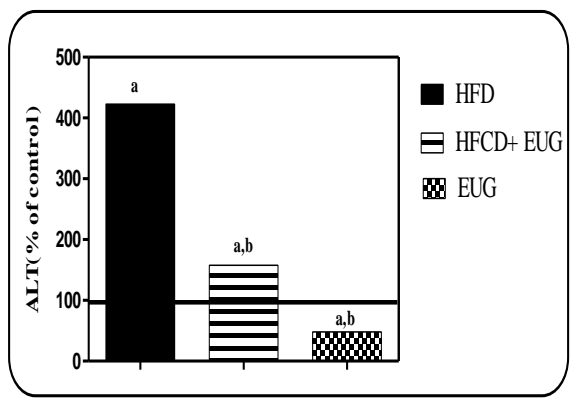

Fig.(2): Effect of EUG on serum level of ALT in rats in rats fed HFCD.

Rats were fed HFCD diet for 8 weeks, HFCD+ EUG, and EUG groups received EUG $(10 \mathrm{mg} / \mathrm{kg})$ orally 3 times weekly for 8 weeks. Data are represented as mean \pm S.D. $(\mathrm{n}=10)$. $\mathrm{a}$, or $\mathrm{b}$ : significant difference from control, or HFCD groups, respectively at $\mathrm{p}<0.05$ using ANOVA followed by Tukey-Kramer as a post-hoc test.

Figure 3: Effect of EUG on serum level of AST in rats fed HFCD:

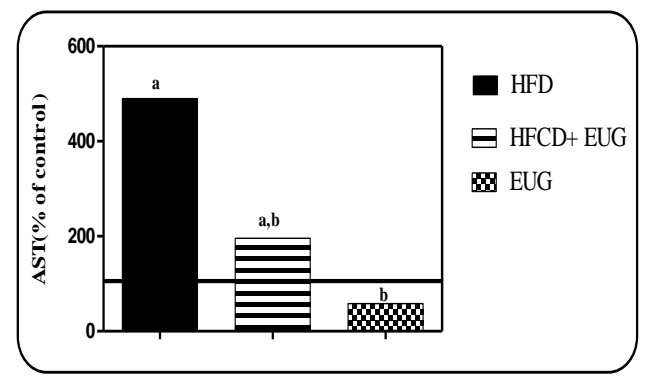

Fig.(3): Effect of EUG on serum level of AST in rats in rats fed HFCD. 
Rats were fed HFCD diet for 8 weeks, HFCD+ EUG, and EUG groups received EUG $(10 \mathrm{mg} / \mathrm{kg})$ orally 3 times weekly for 8 weeks .Data are represented as mean \pm S.D. $(n=10)$. $\mathrm{a}$, or $\mathrm{b}$ : significant difference from control, or HFCD groups, respectively at $\mathrm{p}<0.05$ using ANOVA followed by Tukey-Kramer as a post-hoc test.

\section{Liver histopathological assessment}

Rats fed normal chow diet showed normal hepatic architecture. Rats fed with HFCD diet showed liver steatosis, lobular inflammation, hepatocellular ballooning in diffuse manner all over the hepatocytes as nuclear pyknosis in the hepatocytes surrounding and adjacent the dilated central vein. HFCD+EUG group showed little congestion in the central vein with mild degeneration in hepatocytes (Fig4).

\section{Figure4: Histopathological examination of liver using H\&E stain}

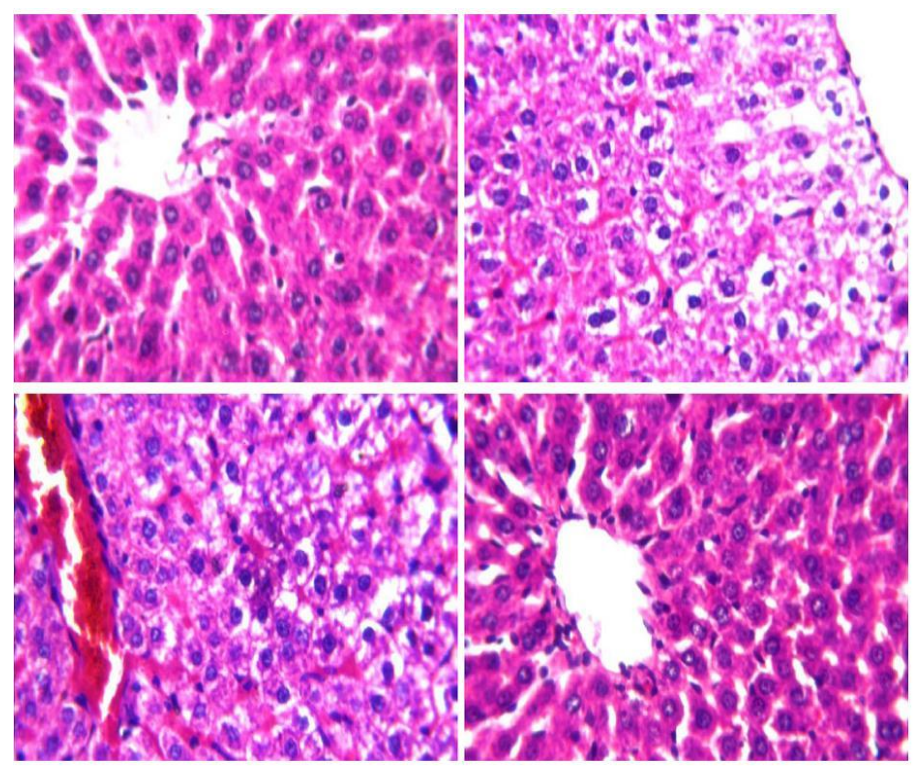

Fig. (4): Photomicrographs of liver sections stained by haematoxylin and eosin $(\times 80)$.

A: section taken from rat in the control showing normal histological structure of the central vein $(\mathrm{CV})$ and surrounding hepatocytes. B: section taken from rat in the HFCD group showing liver steatosis, lobular inflammation, hepatocellular ballooning in diffuse manner all over the hepatocytes. C: section taken from rat in HFCD+EUG group showing little congestion in the central vein with mild degeneration in hepatocytes. D: section taken from rat in EUG only group showing normal histological structure.

\section{Discussion}

NAFLD is a major health public issue due to its high prevalence and it is considered one of the major causes of chronic liver damage (Tilg and Moschen, 2008) . . Recently, EUG is of interest for many recent researchers because of its broad range of pharmacological and biological activities (Jo et al., 2014). It has been used as anti- 
inflammatory agent (Huang $\boldsymbol{e t}$ al., 2015), anti-hyperglycemic agent (Jeong $\boldsymbol{e t}$ al., 2014), and anti-oxidant agent (Gulcin, 2011). The present study was designed to assess the potential protective effect of EUG on IR in NAFLD model in rats.

In the present study, by feeding rats HFCD diet for 8 weeks, rat model of NASH, namely steatosis, was established in HFCD group, as indicated by significant elevation of plasma

ALT and AST activities which reproduce marked liver injury. These results are in alignment with previous study (Yogalakshmi et al., 2010). On the contrary, co-treatment with EUG showed significant decrease in hepatic enzyme levels. Our results were supported by previous study (Srinivasan et al., 2014).

An ideal animal model should displays hepatic histopathologic and pathophysiologic features associated with NASH (Hebbard and George, 2011). .In our model, increase the fat intake showed liver steatosis, lobular inflammation, hepatocellular ballooning in diffuse manner all over the hepatocytes. These results are in alighment with previous studies (Xu et al., 2010; Takahashi et al., 2012). On the other hand, EUG co-treatment ameliorated these changes. Our results are supported by previous study (Venkadeswaran et al., 2014).

\section{Conclusion}

In conclusion, EUG co-treatment significantly ameliorates NAFLD by modulating of liver enzymes (ALT\&AST) levels. Furthermore, Histopathological results supported hepatoprotective role of EUG against HFCD. The results of this study make EUG a promising dietary supplement to improve NAFLD.

\section{REFERENCES}

Arguello G, Balboa E, Arrese $M$ and Zanlungo S (2015). Recent insights on the role of cholesterol in non-alcoholic fatty liver disease. Biochimica et Biophysica Acta (BBA)-Molecular Basis of Disease 1852(9): 1765-1778.

Berlanga A, Guiu-Jurado E, Porras JA and Auguet T (2014). Molecular pathways in non-alcoholic fatty liver disease. Clinical and Experimental Gastroenterology 7: 221-239.

Gulcin I (2011). Antioxidant activity of eugenol: a structure-activity relationship study. Journal of Medicinal Food 14(9): 975-985.

Hebbard L and George J (2011). Animal models of nonalcoholic fatty liver disease. Nature Reviews. Gastroenterology and Hepatology 8(1): 35-44.

Huang X, Liu Y, Lu Y and Ma C (2015). Anti-inflammatory effects of eugenol on lipopolysaccharide-induced inflammatory reaction in acute lung injury via regulating inflammation and redox status. International Immunopharmacology 26(1): 265-271.

Jeong KJ, Quan H-Y, Jo HK, Kim GW and Chung SH (2014). Effects of eugenol on hepatic glucose production and AMPK signaling pathway in hepatocytes and C57BL/6J mice. Fitoterapia 93: 150-162. 
Jirovetz L, Buchbauer G, Stoilova I, Stoyanova A, Krastanov A and Schmidt E (2006). Chemical composition and antioxidant properties of clove leaf essential oil. Journal of Agricultural and Food Chemistry 54(17): 6303-6307.

Jo HK, Kim GW, Jeong KJ, Do YK and Chung SH (2014). Eugenol ameliorates hepatic steatosis and fibrosis by down-regulating SREBP1 gene expression via AMPKmTOR-p70S6K signaling pathway. Biological and Pharmaceutical Bulletin 37(8): 1341-1351.

Kabuto H, Tada M and Kohno M (2007). Eugenol [2-methoxy-4-(2-propenyl) phenol] prevents 6-hydroxydopamine-induced dopamine depression and lipid peroxidation inductivity in mouse striatum. Biological and Pharmaceutical Bulletin 30(3): 423-427.

Kalemba D and Kunicka A (2003). Antibacterial and antifungal properties of essential oils. Current Medicinal Chemistry 10(10): 813-829.

Loria P, Lonardo A and Targher G (2008). Is liver fat detrimental to vessels?: intersections in the pathogenesis of NAFLD and atherosclerosis. Clinical Science 115(1): 1-12.

Nuchuchua O, Saesoo S, Sramala I, Puttipipatkhachorn S, Soottitantawat A and Ruktanonchai U (2009). Physicochemical investigation and molecular modeling of cyclodextrin complexation mechanism with eugenol. Food Research International 42(8): 1178-1185.

Shulman GI (2014). Ectopic fat in insulin resistance, dyslipidemia, and cardiometabolic disease. New England Journal of Medicine 371(12): 1131-1141.

Srinivasan S, Sathish G, Jayanthi M, Muthukumaran J, Muruganathan U and Ramachandran V (2014). Ameliorating effect of eugenol on hyperglycemia by attenuating the key enzymes of glucose metabolism in streptozotocin-induced diabetic rats. Molecular and Cellular Biochemistry 385(1-2): 159-168.

Stienstra R, Saudale F, Duval C, Keshtkar S, Groener JE, van Rooijen N, et al. (2010). Kupffer cells promote hepatic steatosis via interleukin-1 $\beta$-dependent suppression of peroxisome proliferator-activated receptor $\alpha$ activity. Hepatology 51(2): 511522.

Takahashi Y, Soejima Y and Fukusato T (2012). Animal models of nonalcoholic fatty liver disease/nonalcoholic steatohepatitis. World Journal of Gastroenterology 18(19): 2300-2308.

Than NN and Newsome PN (2015). A concise review of non-alcoholic fatty liver disease. Atherosclerosis 239(1): 192-202.

Tilg $H$ and Moschen AR (2008). Insulin resistance, inflammation, and non-alcoholic fatty liver disease. Trends in Endocrinology and Metabolism: TEM 19(10): 371-379.

Venkadeswaran K, Muralidharan AR, Annadurai T, Ruban VV, Sundararajan M, Anandhi R, et al. (2014). Antihypercholesterolemic and antioxidative potential 
of an extract of the plant, Piper betle, and its active constituent, eugenol, in triton WR-1339-Induced hypercholesterolemia in experimental rats. Evidence-Based Complementary and Alternative Medicine 2014.

Xu ZJ, Fan JG, Ding XD, Qiao L and Wang GL (2010). Characterization of high-fat, diet-induced, non-alcoholic steatohepatitis with fibrosis in rats. Digestive diseases and Sciences 55(4): 931-940.

Yogalakshmi B, Viswanathan $P$ and Anuradha CV (2010). Investigation of antioxidant, anti-inflammatory and DNA-protective properties of eugenol in thioacetamideinduced liver injury in rats. Toxicology 268(3): 204-212.

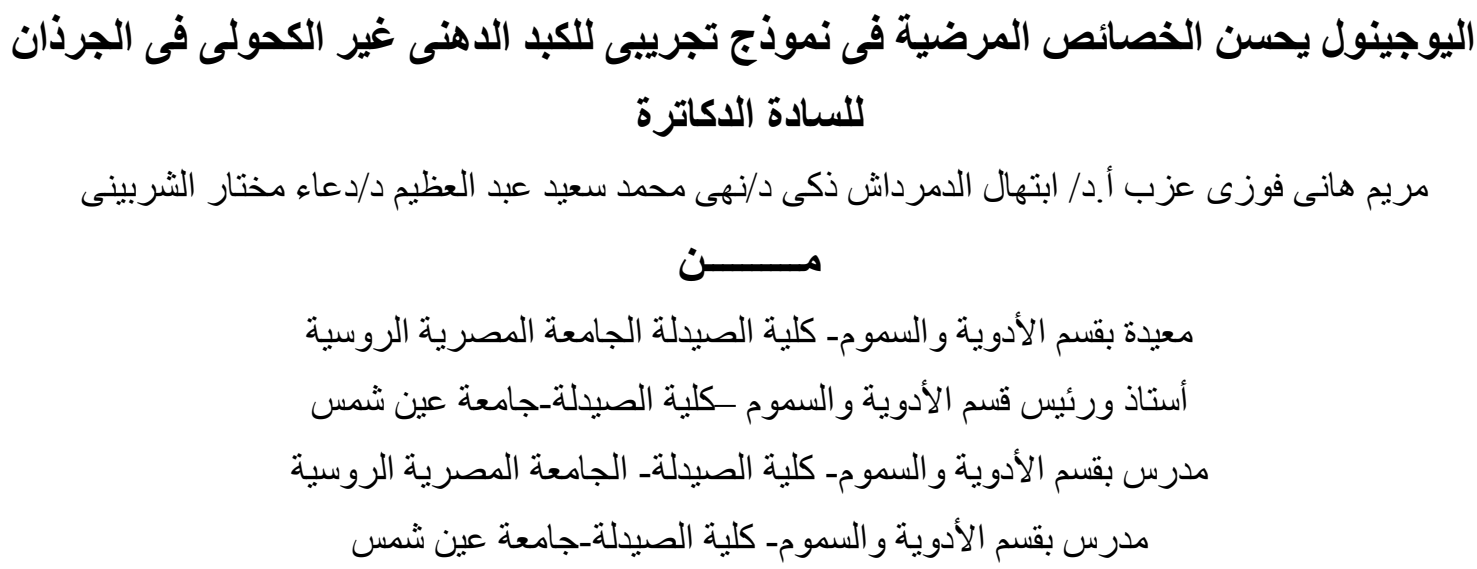

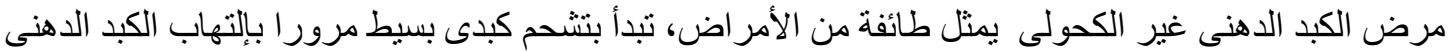

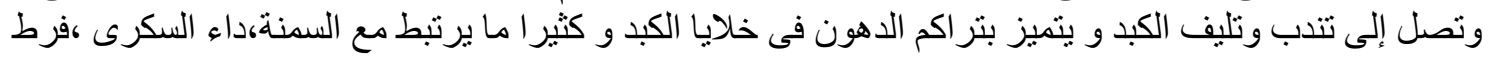

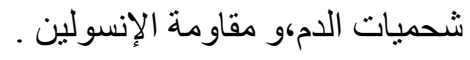

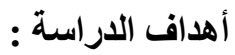
الغرض من هذه الدراسة هو التحقق من التأثير المحتمل لليوجينول فى الكبد الدهنى غير الكحولى . التصميم التجريبى : n

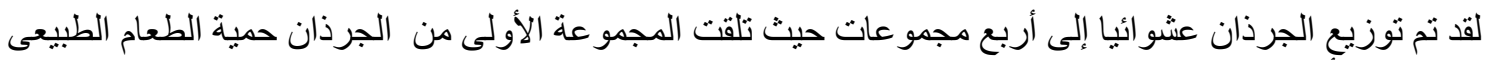

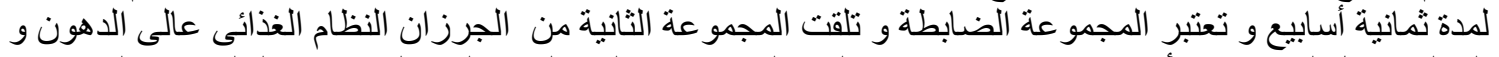

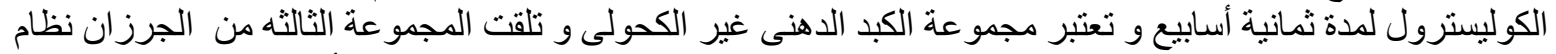

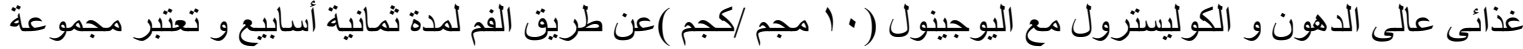

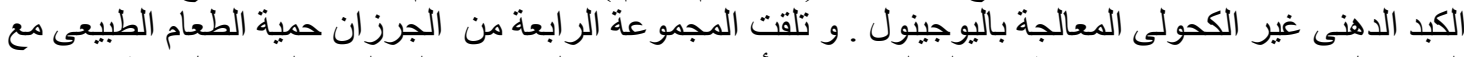

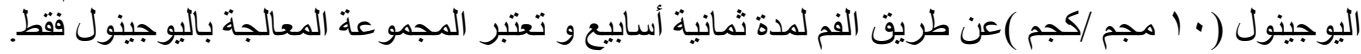

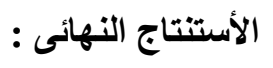

لقد أثتتت النتائج أن لليوجينول القدرة على تحسين مرض الكبد الكبد الدهنى غير الكحولى من خلال تحسين الخصائص

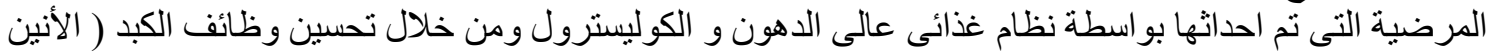

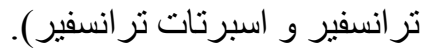

